\title{
Plasmocitoma extramedular del tabique nasal: Reporte de un caso y revisión bibliográfica
}

\author{
Extramedullary plasmacytoma nasal septum: Case report and literature review
}

Daniel Moyano $\mathbf{M}^{1}$, Andrés Mella T², Eduardo Peirano $0^{3}$, Jaime Bermeo $\mathbf{S}^{1}$.

\begin{abstract}
RESUMEN
Los plasmocitomas son tumores malignos caracterizados por proliferación anormal de células plasmáticas monoclonales. Éstos pueden originarse tanto en hueso como en tejidos blandos, denominados plasmocitomas óseos solitarios y plasmocitomas extramedulares (PEM), respectivamente. Estos últimos representan menos del 1\% de toda la patología maligna de cabeza y cuello, sin embargo, el reporte de estos casos resulta provechoso frente a la poca casuística al respecto.

Presentamos el caso de plasmocitoma extramedular del septo nasal en un varón de 74 años con dificultad respiratoria nasal progresiva y frecuente epistaxis del lado izquierdo. A la especuloscopía nasal se observó en el vestíbulo una elevación tumoral de superficie lisa, grisácea, sésil. La biopsia mostró que era un plasmocitoma. Estudios posteriores descartaron la presencia de mieloma múltiple. Esto confirmó el diagnóstico de PEM. La masa fue completamente disecada, se indicó radioterapia y el paciente no tuvo adherencia al tratamiento. Sobre el mismo lecho al año siguiente reaparece lesión de similares características.

Conclusión: Plasmocitoma extramedular del tabique nasal es una entidad rara, de una larga historia natural y que representa un desafío diagnóstico y terapéutico. Dependiendo de la resecabilidad de la lesión, la terapia combinada mediante cirugía y radioterapia es el tratamiento de elección.
\end{abstract}

\begin{abstract}
Plasmacytomas are malignant tumors characterized by abnormal proliferation of monoclonal plasma cells. They may originate in bone and soft tissue, called solitary bone plasmacytoma and extramedullary plasmacytomas (EMP) respectively. The latter represents less than $1 \%$ of all malignant disease of the head and neck, however, the report of these cases it is useful to cope with the shallow casuistics thereon.
\end{abstract}

1 Médico Servicio Otorrinolaringología, Hospital Carlos Van Buren.

2 Interno de Medicina, Universidad de Valparaíso.

3 Médico Cirujano, Hospital Quilpué, Universidad de Valparaíso.

Recibido el 23 de abril de 2015. Aceptado el 2 de julio de 2016. 
We report a case of EMP of the nasal septum in a man of 74 years with progressive nasal breathing difficulties and frequent epistaxis on the left side. A nasal speculoscopy was observed in tumor lift lobby smooth, gray, sessile surface. The biopsy showed it was a plasmacytoma. Subsequent studies reject the presence of multiple myeloma. This confirmed the diagnosis of EMP. The mass was completely dissected, radiation therapy is indicated and the patient had no adherence. Upon the same place a similar injury returns.

Conclusion: Septum extramedullary plasmacytoma is a rare entity of a long natural history and represents a diagnostic and therapeutic challenge. Depending on the resectability of the lesion, combination therapy with surgery and radiation therapy is the treatment of choice.

\section{INTRODUCCIÓN}

Los plasmocitomas son tumores malignos caracterizados por una proliferación subepitelial de células plasmáticas monoclonales anormales. Éstos se clasifican dependiendo del origen del tejido afectado. Los que se originan en el hueso se llaman plasmocitomas óseos solitarios. Los que se inician en tejidos blandos se denominan plasmocitomas extramedulares (PEM) $)^{1,2}$. Cualquiera de estos tumores puede constituir un foco primario o formar parte de la evolución sistémica del mieloma múltiple 3 . PEM es una entidad de etiología desconocida y poco frecuente pero, cuando se presenta, suele afectarse con más frecuencia en el área otorrinolaringológica ${ }^{4}$. Fue descrita la primera vez en 1905 por Schridde ${ }^{5}$ y en 1999 Alexiou y cols realizaron una revisión de todos los casos comprendidos entre 1905 a 1997, que incluyó a 869 pacientes, de los cuales 714 (82,2\%) tuvieron compromiso de la vía aerodigestiva alta. De éstos el $43,8 \%$ correspondían a cavidad nasal y senos paranasales, $18,3 \%$ a nasofaringe, $17,8 \%$ a orofaringe y $11,1 \%$ a laringe ${ }^{6}$. Otros sitios de menor porcentaje incluyen la lengua, glándulas salivales menores, tiroides, paratiroides, órbita y hueso temporal 7 . Su incidencia es de 3,5/100.000 al año $0^{6}$, es 4 veces más frecuente en hombres que en mujeres ${ }^{7}$ y el $95 \%$ de los casos ocurre sobre los 40 años de edad, principalmente entre la sexta y séptima década de vida ${ }^{8}$. El $80 \%-90 \%$ se presenta en cabeza y cuello siendo menos del $1 \%$ de las neoplasias en esta área ${ }^{4}$. PEM puede manifestarse como una elevación tumoral de superficie lisa 0 como una masa subepitelial de color rosado, gris, o rojizo; únicos, múltiples, polipoideos sésiles 0 pediculados, difusos o ulcerados ${ }^{9}$. Es un tumor destructivo que aparte de tener recurrencia loca ${ }^{8,9}$ tiene la capacidad de diseminarse hacia linfonodos regionales en el 8\%-32\%, generar metástasis ${ }^{10} \mathrm{y}$ progresar hacia mieloma múltiple. Sus manifestaciones clínicas pueden darse como masa nasal, aumento de volumen, dolor facial, obstrucción de la vía aérea, epistaxis, rinorrea, proptosis, disfagia o disfonía9. La rareza de este tumor y su larga historia natural constituye un gran desafío diagnóstico ${ }^{11}$, en el cual se deben realizar estudios histológicos que deben complementarse con inmunohistoquímica ${ }^{12,13}$. Su histología se describe como una proliferación monomórfica subepitelial de plasmocitos que pueden ser diferenciados 0 anaplásicos, con potencialidad de tener infiltración subyacente. Para el tratamiento y la sobrevida del paciente es fundamental diferenciarla del mieloma múltiple y del plasmocitoma óseo solitario ${ }^{14}$ mediante estudios y seguimiento con perfil bioquímico completo, calcio, proteínas plasmáticas, electroforesis de proteínas séricas, proteínas de Bence Jones urinarias, estudio radiográfico completo del esqueleto, hemograma, mielograma, y de ser necesario, una biopsia de médula ósea ${ }^{12,15}$. El manejo terapéutico del PEM es un tema controversial, sin embargo se ha visto que las células plasmáticas tumorales tienen una gran sensibilidad a la radioterapia, aceptándose a ésta como su tratamiento de elección ${ }^{16,17}$. Se ha visto también buenos resultados en el control local con la cirugía, siempre y cuando sea posible su resección por su tamaño y ubicación $n^{6}$.

A continuación se presentará un caso de PEM del septo nasal. Como los PEM son de baja prevalencia, el manejo diagnóstico y terapéutico se 
ve facilitado gracias a la experiencia basada en la casuística de reportes individuales previos.

\section{CASO CLÍNICO}

Paciente de sexo masculino de 74 años, con antecedentes de diabetes mellitus 2 insulino requirente, enfermedad renal crónica etapa III, dislipidemia, hipertensión arterial crónica, insuficiencia cardíaca crónica con hemibloqueo anterior izquierda-bloqueo aurículo ventricular de primer grado y sobrecarga ventricular. Sin antecedentes de alergias.

Consultó hace más de un año al servicio de otorrinolaringología por cuadro de 3 meses de evolución caracterizado por obstrucción en fosa nasal izquierda acompañado de epistaxis intermitente en el último mes. Refiere ortopnea, disnea paroxística nocturna y capacidad funcional II. Al examen físico destaca obesidad mórbida, abdomen globoso. Extremidades inferiores levemente edematosas, bien perfundidas a distal.

Al examen físico otorrinolaringológico destacó tumor en vestíbulo fosa nasal izquierda, proptosis ojo izquierdo, sin compromiso de agudeza visual ni motricidad ocular. Exámenes de laboratorio se destacaron: glicemia $151 \mathrm{mg} / \mathrm{dl}$, leucocitos en plasma $13.750 / \mathrm{mm}^{3}$, creatinina plasmática 1,73 $\mathrm{mg} / \mathrm{dl}$. Se decidió completar estudio con tomografía computarizada contrastada de cavidades paranasales (Figura 1) la cual reveló desviación

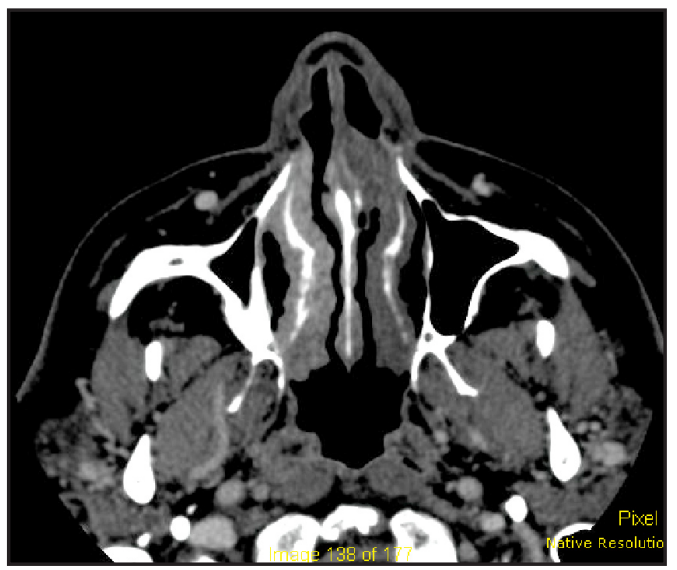

Figura 1. TAC cavidades paranasales corte axial que muestra masa tumoral en la mucosa del tabique de la fosa nasal izquierda. dextroconvexa del tabique nasal, pequeño espolón óseo y lesión heterogénea de la mucosa del tabique fosa nasal izquierda, ubicado a nivel del tercio medio y posterior del cartílago cuadrangular, respetando columela y vómer. De diámetros de 18 x 19 mm, impregnándose levemente con contraste y contactando en forma estrecha con cornete inferior izquierdo.

Se realizó una biopsia excisional en tabique con sedación y anestesia local, resecando fragmento irregular color pardo, moderadamente firme, de superficie lisa y con estructura sólida que midió $3,2 \times 2 \times 0,9 \mathrm{~cm}$. Al estudio anatomopatológico bajo tinción H-E (Figura 2) se informó una neoplasia estromal compuesta de plasmocitos bien diferenciados, sin atipia.

Además se presentaron depósitos de amiloide destacados a la tinción de rojo congo (Figuras 3 y 4), que representaron un tercio del volumen tumoral.

Se diagnosticó como plasmocitoma con amiloidosis localizada. Imnunohistoquímica positiva para cadenas livianas. Se planteó el diagnóstico de plasmocitoma extramedular, derivándose al servicio de hematología para completar el estudio con la finalidad de descartar un mieloma múltiple. Para ello realizaron una serie de exámenes que incluyeron mielograma tomado del cuerpo esternal, electroforesis de proteínas plasmáticas, cuantificación de inmunoglobulinas, hemograma, pruebas de coagulación y perfil bioquímico.

El mielograma se informó como médula ósea de características normales. Electroforesis de pro-

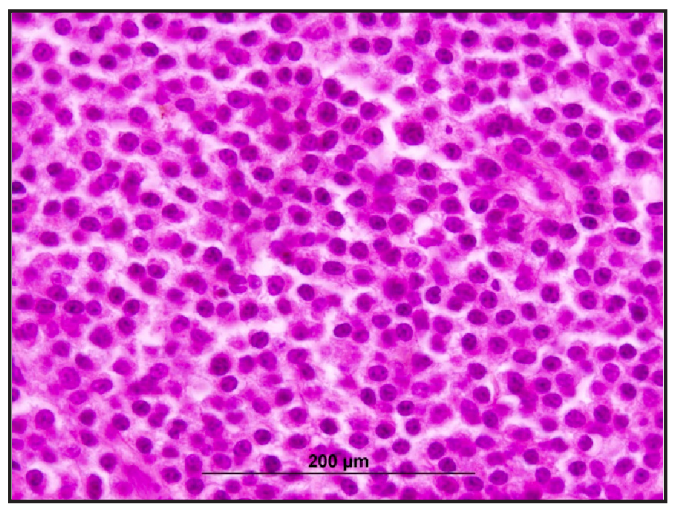

Figura 2. Biopsia tumor tabique nasal bajo visión microscópica que muestra plasmocitos bien diferenciados lo que da el diagnóstico de plasmocitoma. Tinción H-E x 100. 


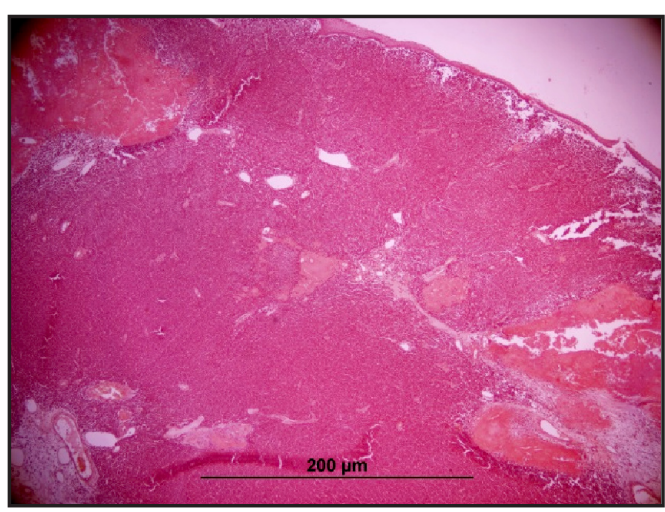

Figura 3. Biopsia tumor tabique nasal. Tinción rojo congo x 5 , el cual permite destacar extensos depósitos de amiloide dentro de la tumoración infiltrada de plasmocitos bien diferenciados lo que da el diagnóstico de plasmocitoma con amiloidosis localizada.

teínas plasmáticas sólo destacó hipoproteinemia de $6,28 \mathrm{~g} / \mathrm{dl}$ (VN: $6,4-8,3 \mathrm{~g} / \mathrm{dl}$ ) e hipoalbuminemia de 2,98 g/dl (VN: 3,0-5,0 g/dl) sin relevancia clínica; el resto de las proteínas dentro de rangos normales. Cuantificación de inmunoglobulinas dentro de parámetros normales. En el hemograma destaca una leucocitosis de $16.090 / \mathrm{mm}^{3}$, sin anemia y VHS dentro de rangos normales. Pruebas de coagulación normales. El perfil bioquímico solo destaca una creatininemia de 1,88 mg/dl $(0,72-1,25 \mathrm{mg} / \mathrm{dl})$.

Se confirmó el diagnóstico de plasmocitoma extramedular de tabique nasal al descartarse la existencia de mieloma múltiple.

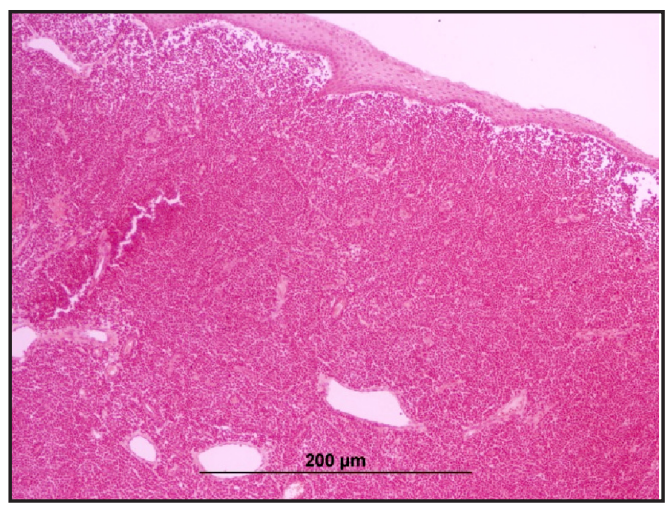

Figura 4. Biopsia tumor tabique nasal. Depósitos de amiloide en lagunas más pequeñas dentro del infiltrado plasmocitario. Tinción rojo congo x 10 .

Se indicó radioterapia local de 50 Gray, el cual no se realizó por contraindicaciones técnicas, debido a que su ortopnea no le permitió soportar la posición en decúbito dorsal. Se hicieron controles hematológicos cada 4 meses sin cambios significativos. Al año siguiente nuestro paciente consultó al servicio de otorrinolaringología con una lesión en placa úlcero infiltrativa sobre lecho fibrinoso (Figura 5), en la misma zona de implantación anterior, de $1 \mathrm{~cm}$ de diámetro y $1 \mathrm{~mm}$ de espesor, de bordes no definidos. Se procede a realizar biopsia excisional la cual concluye restos de tejido amiloídeo, sin evidencia de plasmocitoma.

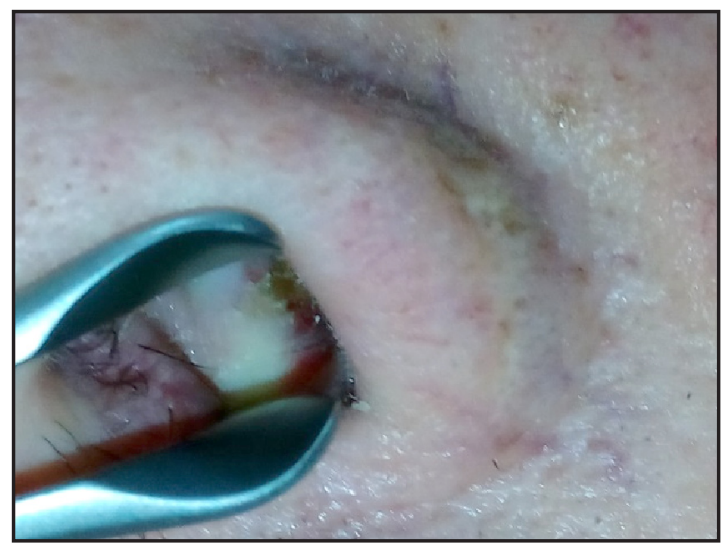

Figura 5. Lesión en placa úlcero infiltrativa sobre lecho fibrinoso correspondiente a la misma zona donde se implantó la lesión anterior. 


\section{DISCUSIÓN}

Frente a un plasmocitoma, lo primero que se debe reconocer es que si se trata de un tumor primario (PEM) 0 de una metástasis (mieloma múltiple) ${ }^{9}$, lo que ocurre en 1 de cada 40 pacientes ${ }^{18}$. Este tumor se caracteriza por su lenta historia natural, síntomas atípicos y reconocimiento tardío ${ }^{11}$. No basta diagnosticarlo sólo por tomografía computarizada y RNM debido a su falta de especificidad $^{19}$. Tampoco basta con estudios morfológicos de la pieza operatoria, pues se requiere de estudios complementarios mediante pruebas de inmunohistoquímica que demuestra la naturaleza monoclonal de las células plasmáticas, además de descartar otras malignidades como el carcinoma indiferenciado ${ }^{12,13}$. Una vez confirmado el diagnóstico de plasmocitoma, la segunda etapa diagnóstica consiste en descartar el compromiso sistémico de un mieloma múltiple cuyas características clínicas corresponden a tumoraciones, anemia, insuficiencia renal, infecciones, alteraciones de la coagulación, dolor óseo y alteraciones neurológicas ${ }^{20}$. El estudio se realiza con hemograma, mielograma, biopsia de médula ósea, perfil bioquímico completo, calcio, proteínas séricas, electroforesis de proteínas plasmáticas y urinarias, y radiografía para descartar lesiones líticas ${ }^{12,15}$. En nuestro caso, su nefropatía era diabética. Su estudio no incluyó biopsia de médula ósea debido a que el mielograma fue concluyente, con plasmocitos maduros de solo $3 \%$, presencia de las 3 series hematopoyéticas con características morfológicas normales, médula ósea de características normales dentro del contexto de un paciente que no tiene anemia ni VHS sobre 100 $\mathrm{mm} /$ hora. La cuantificación de inmunoglobulinas y electroforesis de proteínas plasmáticas resultaron dentro de parámetros normales, por lo que se confirmó el PEM.

El tratamiento de PEM localizado en cabeza y cuello es controvertido. Algunos autores defienden la radioterapia mientras otros prefieren la cirugía. Cabe señalar que las células tumorales plasmáticas son sensibles a la radiación, razón por la cual la radioterapia es el tratamiento aceptado. Sin embargo, cuando existe un abundante depósito amiloide dentro de la tumoración, la radioterapia no siempre reducirá el tamaño del tumor ${ }^{21-23}$. Ésta es la razón por la que la mayoría de los clínicos recomiendan un tratamiento combinado que incluya cirugía más radioterapia en el manejo del PEM de la cavidad nasal ${ }^{23,24}$. Existe la evidencia en los estudios de Alexiou y cols mostrando que esta combinación da mejores resultados. En sus revisiones de 714 casos de PEM de la vía aerodigestiva alta entre Ios años 1905-1997, encontraron que la sobrevida promedio sin recurrencia en pacientes con terapia combinada fue mayor a 300 meses, en aquellos que tuvieron solo radioterapia 144 meses y quienes recibieron solo cirugía 156 meses $^{6}$. La posibilidad de intervención quirúrgica depende de la ubicación y tamaño del tumor.

Cabe señalar que en nuestro caso, su histología destacó un infiltrado amiloide, lo que reduciría el éxito de la radioterapia por sí sola. Sin embargo, nuestro paciente no soportaba posición en decúbito dorsal, causal por la que no tuvo adherencia a tratamiento coadyuvante con radioterapia.

\section{CONCLUSIONES}

Plasmocitoma extramedular del tabique nasal es una entidad infrecuente, de una larga historia natural y que representa un desafío diagnóstico y terapéutico porque debe excluirse la presencia de mieloma múltiple. Consiste en un tumor submucoso bien delimitado que puede ser agresivo localmente. Este plasmocitoma puede corresponder a una metástasis de un mieloma múltiple 0 , siendo éste un PEM, puede evolucionar hacia un mieloma múltiple. De ahí la necesidad de manejo y seguimiento por equipo multidisciplinario. Terapia combinada mediante cirugía y radioterapia es el tratamiento de elección dado a su mejor pronóstico, siempre y cuando la resecabilidad de la lesión sea posible. 


\section{BIBLIOGRAFÍA}

1. Batsakis JG, Medeiros Jl, Luna MA, El-Naggar AK. Plasma cell dyscrasias and the head and neck. Ann Diagn Pathol 2002; 6: 129-40.

2. Ronald B. Kuppersmith. Extramedullary plasmocytoma of the head \& neck. Rounds Archive al Baylor March 28, 1996.

3. Callihan TR, Holbert JM, Jr. Neoplasm of terminal B cell differentiation. Norwalk: Appleton Century Crafts; 1983; 169-268.

4. Lippincott Williams \& Wilkins. Head and Neck Surgery Otolaryngology. $2^{\text {nd }}$ Edition. 1998.

5. Ronald B. Kuppersmith. Extramedullary plasmocytoma of the head \& neck. Rounds Archive al Baylor. March 28, 1996.

6. Alexiou C, Kau R, Dietzfelbinger H, Kremer M, Spiel J, Schratzenstaller B et al. Extramedular plasmacytoma: tumor occurrence and therapeutic concepts. Cancer 1999; 85: 2305-14.

7. Wax MK, Yun KJ, OMAR RA. Extramedullary plasmacytomas of the head and neck. Otolaryngol Head Neck Surg 1993; 109: 877-85.

8. Kapadia SB, Desal U, Cheng VS. Extramedullary plasmacytoma of the head and neck. A clinicopathologic study of 20 cases. Medicine (Baltimore) 1982; 61: 317-29.

9. Wiltshaw E. The Natural history of extramedullary plasmacytoma and its relation to solitary myeloma of bone and myelomatosis. Medicine 1976; 55: 217-38.

10. Gonzales J, Ghufoor K, Sandhu G, Thorpet P, HAdLeY J. Primary plasmacytoma of the parotid gland: a case resport and review of the literature. Journal of Laryngology and Otology 1998; 112: 179-81.

11. Berlic B, Mitrovic S, Arsenijevic S, Erdevicki L, Stojanovic J, Stojanovic S, et al. Nasal septum extramedullary plasmacytoma. Vojnosanit Pregl 2013; 70: 221-4.

12. Miller FR, Lavertu P, Wanamaker JR, Bonafede J, Wood BG. Plasmacytomas of the head and neck. Otolaryngol Head Neck Surg 1998; 119: 614-8.
13. Komisar A, Schetman F, DaSilva M, Ioachim $H$, BLAUGRUnd SM. The histopathologic diagnosis of head and neck tumors by special stains. Ear Nose Throat J 1989; 68: 702, 705-6, 709-12.

14. Gonzales J, Ghufoor K, Sandhu G, Thorpet P, HADLEY J. Primary plasmacytoma of the parotid gland: a case resport and review of the literature. Journal of Laryngology and Otology 1998; 112 : 179-81.

15. Hotz MA, Schwaab G, Bose J. Munck JN. Extramedullary solitary plasmacytoma of the head \& neck. A clinicopathological study. Ann Otol Rhinol Laryngol 1999; 108: 495-500.

16. Jyothirmay R, GangadhaRan VP, Nair MK, Rajan B. Radiotherapy in the treatment of solitary plasmacytoma. Br J Radiol 1997; 70: 511-6.

17. Mendenhall CM, Thar TL, Mıllior RR. Solitary plasmocytoma of bone and soft tissue. Int $J$ Radiat Oncol Biol Phys 1980; 6: 1497-501.

18. Anderson KC, Park EK, Bates MP, Leonard RC, Hardy R, Schlossman SF et al. Antigens on human plasma cells identified by monoclonal antibodie. J Inmunol 1983; 130: 1132-8.

19. Ching AS, Khoo JB, Chong VF. CT and MR imaging of solitary extramedullary plasmacytoma of the nasal tract. AJNR Am J Neuroradiol 2002; 23 : 1632-6.

20. Kyle R, Rajkumar S. Multiple Myeloma. The New England Journal of Medicine 2004; 351: 186073.

21. LeBowitz RA, MorRIs L. Plasma cell dyscrasias and amyloidosis. Otolaryngol Clin North Am 2003; 36: 747-64.

22. Hidaka H, Ikeda K, Oshima T, Ohtani H, Suzuki $H$, TAKASAKA T. A case of extramedullary plasmacytoma arising from the nasal septum. $J$ Laryngol Otol 2000; 114: 53-5.

23. WINDFUHR JP, 0TT G. Extramedullary plasmacytoma manifesting as a palpable mass in the nasal cavity. Ear Nose Throat J 2002; 81: 110-4.

24. Navarrete ML, Quesada P, Pellicer M, Ruiz C. Extramedullary nasal plasmacytoma. J Laryngol Otol 1991; 105: 41-3.

Dirección: Daniel Moyano Miranda

Hospital Carlos van Buren, Valparaiso, Chile

E mail: danimoyano@gmail.com 